\title{
Diagnosing non-hepatocellular carcinoma malignancies on CT/MRI and contrast enhanced ultrasound: the Liver Imaging Reporting and Data System approach
}

\author{
Kathryn J. Fowler ${ }^{1}$, Guilherme Moura Cunha', Tae Kyoung Kim² \\ 'Department of Diagnostic Radiology, University of California at San Diego, San Diego, CA 92130, USA. \\ 2University of Toronto, Joint Department of Medical Imaging, University Health Network/Mount Sinai Hospital/Women's \\ College Hospital, Toronto M5G 2N2, Canada.
}

Correspondence to: Dr. Kathryn J. Fowler, Department of Diagnostic Radiology, University of California at San Diego, 200 West Arbor Drive \#8756, San Diego, CA 92130, USA. E-mail: k1fowler@health.ucsd.edu

\begin{abstract}
How to cite this article: Fowler KJ, Cunha GM, Kim TK. Diagnosing non-hepatocellular carcinoma malignancies on CT/MRI and contrast enhanced ultrasound: the Liver Imaging Reporting and Data System approach. Hepatoma Res 2020;6:19. http://dx.doi.org/10.20517/2394-5079.2020.21
\end{abstract}

Received: 2 Mar 2020 First Decision: 16 Apr 2020 Revised: 20 Apr 2020 Accepted: 24 Apr 2020 Published: 28 Apr 2020

Science Editor: Yuko Kono Copy Editor: Jing-Wen Zhang Production Editor: Tian Zhang

\begin{abstract}
The Liver Imaging Reporting and Data System (LI-RADS) provides a stepwise algorithmic approach that is proven to be highly accurate in diagnosing hepatocellular carcinoma (HCC) in patients at risk. An essential and early step in the algorithm is the diagnosis of malignancies other than HCC, such as cholangiocarcinoma and combined tumors, by application of LR-M features and criteria. The LR-M category captures most non-HCC malignancies and some atypical HCCs. The exclusion of non-HCC malignancies is important for maintaining the high specificity of the LR-5, definite HCC category. This review provides an overview of the approach to diagnosing non-HCC malignancies using LI-RADS CT/MRI and contrast enhanced ultrasound algorithms.
\end{abstract}

Keywords: Magnetic resonance imaging, computer tomography, contrast-enhanced ultrasound

\section{INTRODUCTION}

According to most major liver societies, screening/surveillance imaging is recommended for patients with cirrhosis to detect early stage hepatocellular carcinoma (HCC). While HCC is the most common primary liver malignancy, cirrhosis and chronic viral hepatitis also place patients at risk for non-HCC malignancies such as intrahepatic cholangiocarcinoma (iCCA) and combined hepatocellular-cholangiocarcinomas (cHCC-CCA $)^{[1,2]}$. As a result, these non-HCC malignancies are occasionally found on surveillance imaging

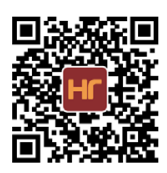


in high-risk patients. There are substantial differences in the treatment strategy and prognosis between HCC and non-HCC malignancies and it is critical to differentiate between the two.

The Liver Imaging Reporting and Data System (LI-RADS) is endorsed by the American College of Radiology and the American Association for the Study of Liver Diseases ${ }^{[3]}$. LI-RADS provides the most comprehensive guidance for imaging in at risk patients, with imaging algorithms covering ultrasound screening/surveillance, diagnosis on CT/MRI, diagnosis on contrast enhanced ultrasound (CEUS), and treatment response on CT/ MRI. This manuscript will provide an overview of the imaging appearances and LI-RADS approach to diagnosing iCCA and cHCC-CCA in at risk patients, both on CT/MRI and on CEUS.

\section{CT/MRI}

\section{Imaging appearance of iCCA and CHCC-CCA}

On CT and MRI, both iCCA and HCC may show arterial phase hyperenhancement (APHE) and washout. It is the morphology of the enhancement during the dynamic postcontrast phases that helps differentiate between them. These differences in morphology are likely attributable to different histological composition. Intrahepatic mass forming cholangiocarcinomas frequently show rim APHE, peripheral washout appearance and often delayed central enhancement ${ }^{[4]}$. It is hypothesized that this targetoid pattern on dynamic imaging may reflect the peripheral cellularity, central necrosis and dense fibrous stroma seen in iCCA ${ }^{[5]}$. This same histological constitution likely also explains the targetoid appearance seen on MRI diffusion weighted and hepatobiliary phase imaging that has been described in $\mathrm{iCCA}^{[6-10]}$. Other ancillary features reported in iCCA include capsular retraction, peripheral biliary duct dilation, and central T2 hypointensity.

On the contrary, HCC tends to have nonrim APHE, nonperipheral washout appearance and delayed enhancing capsule (i.e., major features). These features are likely attributable to the changes during hepatocarcinogenesis that result in the development of unpaired arteries and loss of portal tracts ${ }^{[5]}$. Unlike iCCA, HCC may be encapsulated by a true fibrous capsule or compressed liver parenchyma, which may explain the delayed enhancing "capsule" described on imaging.

Small iCCAs can have overlaps in imaging appearance with $\mathrm{HCC}^{[11,12]}$. Small iCCAs have a greater propensity for showing nonrim APHE and nonperipheral washout appearances, potentially due to a preservation of portal tract architecture and the lack of significant central necrosis in smaller, early stage lesions ${ }^{[5,13]}$. Atypical appearances and overlap with HCC may be more commonly observed in patients with risk factors for HCC such as background liver cirrhosis ${ }^{[14]}$. The reason for this is not entirely understood but could relate to alterations in background liver blood supply (e.g., in the setting of cirrhosis, the liver receives more arterial supply and relatively less portal venous supply).

\section{LI-RADS approach to diagnosis on CT/MRI}

The LI-RADS CT/MRI diagnostic algorithm provides a step-wise approach for arriving at a highly specific diagnosis of HCC. The intention of the algorithm is to achieve a greater than 95 percent positive predictive value of the LR-5 (definite HCC) category for the diagnosis of HCC. The philosophy behind this approach rests in the fact that liver transplantation is considered the optimal cure for patients in the United States and Canada with cirrhosis and early stage HCC. In this context, definitive imaging diagnosis of HCC is sufficient for assigning priority on the transplantation waitlist and as such, demands a near zero false positive rate.

The algorithm begins with assessment of imaging adequacy and assignment of LR-NC (not categorizable) if imaging is not adequate to narrow in on a final diagnostic category. Second, the radiologist should determine if there is advanced disease, such as tumor in vein (LR-TIV). The next step involves identifying definitely or probably benign entities (e.g., perfusional shunts or hemangiomas) and assigning them an LR-1 or LR-2 categorization. Next, the radiologist is tasked with identifying possible or probable non-HCC malignancy 

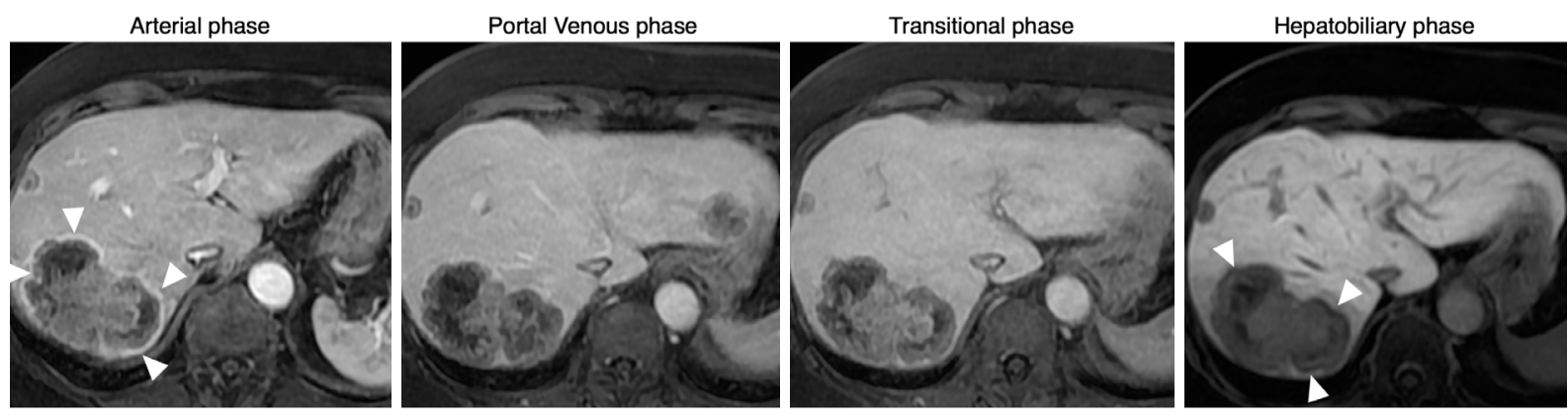

Figure 1. Gadoxetate enhanced MRI: 55 year-old male with chronic hepatitis B infection. A targetoid observation (65 mm) exhibits features of LR-M: rim arterial phase hyperenhancement and peripheral washout (arrowheads). After biopsy, the lesion was diagnosed as liver metastasis from colorectal cancer
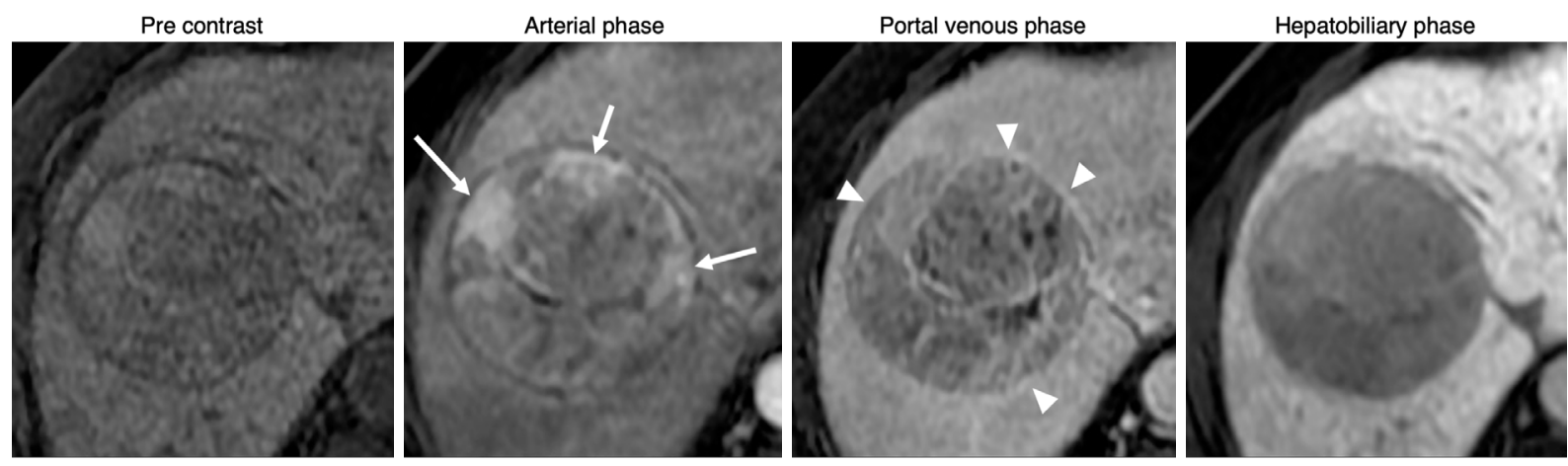

Figure 2. Gadoxetate enhanced MRI: 65 year-old male with chronic hepatitis $C$ cirrhosis. A 60-mm observation exhibits features of LR-5: non rim arterial phase hyperenhancement (arrows), washout and capsule (arrowheads) indicating definite hepatocellular carcinoma. Ancillary features seen are a mosaic architecture and hepatobiliary phase hypointensity

(LR-M). This task will be expanded on below. Once TIV, benign entities, and other malignancies have been excluded in a step-wise fashion, the radiologist should be left with solid hepatocellular nodules that are further classified as intermediate (LR-3), probable (LR-4) or definite HCC (LR-5), according to the presence and number of major features. In LI-RADS, the major features include size, APHE, washout appearance, delayed enhancing capsule, and threshold growth.

The LR-M category is a very important step in the algorithm, as non-HCC malignancies must be considered to avoid false positives in diagnosing LR-5. The features of LI-RADS LR-M primarily reflect the features of iCCA as described above: targetoid patterns in dynamic enhancement, diffusion weighted imaging, and hepatobiliary phase appearances ${ }^{[15]}$. Figure 1 demonstrates a typical non-HCC malignancy with LR-M targetoid features. For comparison, Figure 2 shows a typical LR-5, definite HCC, on MRI. The presence of any targetoid feature is sufficient for LR-M categorization, even if the observation has some features of HCC. Additional features may be applied in malignant observations that do not meet LR-5 or TIV criteria: marked diffusion restriction, necrosis, and infiltrative appearances. Beyond the LR-M features described here, there are ancillary features that favor malignancy in general (e.g., subthreshold growth, corona appearance, hepatobiliary phase hypointensity); however, these ancillary features are not, by themselves, sufficient to categorize an observation as LR-M.

LI-RADS recognizes that imaging features and morphologies are subjective in nature. To help radiologists, there are tie breaking rules both for features and final diagnostic categories. When in doubt, the radiologist should refer to the category or feature that is less specific for HCC. For example, if there is a question of whether APHE is rim or nonrim, the radiologist should assign rim APHE. Likewise, if there is doubt between LR-5 vs. LR-M, the radiologist should assign LR-M. 


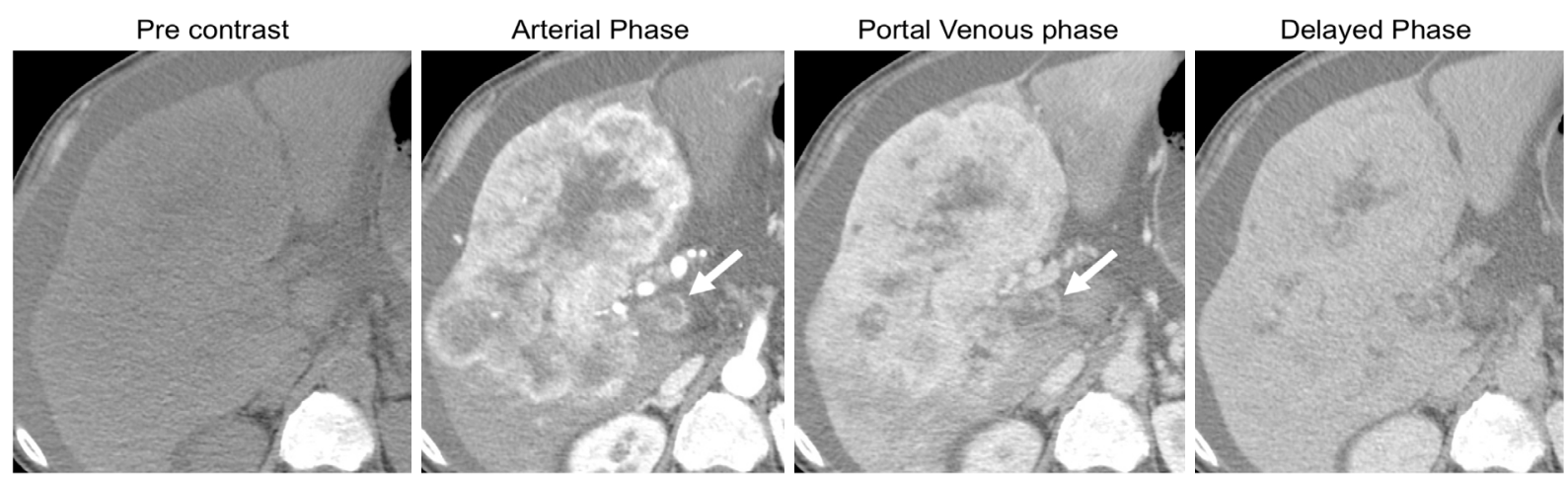

Figure 3. Contrast enhanced $\mathrm{CT}$ : 70 year-old male with chronic hepatitis $\mathrm{B}$. A $120-\mathrm{mm}$ mass in the right liver lobe exhibits a targetoid appearance in the arterial phase with central progressive enhancement in the portal venous and delayed phases, indicating a LR-M observation. There are also features of tumor in vein (arrows), shown as portal vein expansion with heterogeneous contrast enhancement. Biopsy results indicated a cholangiocarcinoma

\section{How accurate is CT/MRI LI-RADS in differentiating iCCA from HCC?}

The LI-RADS approach accurately assigns most non-HCC malignancies, including iCCA, to the LR-M category $^{[16-19]}$. It is important to note that unlike LR-5, the LR-M category is aimed at high sensitivity rather than high specificity for non-HCC malignancies. Hence, it is not unexpected that some atypical HCCs will be categorized as LR-M. In a systematic review and meta-analysis of 17 journal articles published by van der Pol et al. ${ }^{[20]}$, the vast majority (93\%CI: $87 \%-97 \%$ ) of lesions classified as LR-M were malignant and about two thirds were non-HCC malignancies. In other words, about $1 / 3$ of LR-M lesions were in fact atypical HCC. Atypical HCC may represent cirrhotomimetic, sarcomatoid, macrotrabeular massive, schirrhous or other variants that may show LR-M features ${ }^{[21-24]}$. Emerging data suggest that LR-M categorization may provide prognostic information for HCC and cHCC-CCA tumors, with shorter disease free progression and overall survival compared to tumors that are categorized as LR- $5^{[25]}$. Figure 3 shows an aggressive LR-M observation with signs of TIV.

\section{CEUS}

\section{Imaging appearance}

On CEUS, both iCCA and HCC often show APHE and washout. CEUS can detect APHE of liver observations more sensitively than CT/MRI due to real-time assessment of arterial phase enhancement. Therefore, earlier studies raised a concern for misdiagnosis of iCCA as HCC as both often show APHE and washout $^{[26]}$. However, more recent studies have consistently demonstrated that CEUS reliably differentiates iCCA from HCC if the pattern of APHE and the degree and timing of washout are considered ${ }^{[27-30]}$. On CEUS, iCCAs commonly show rim APHE which is uncommon in HCC. Early washout within $60 \mathrm{~s}$ after contrast injection and marked washout are consistently demonstrated in iCCA ${ }^{[31,32]}$. Very early washout within the arterial phase time frame, which is frequently seen in iCCA on CEUS, may explain their frequent arterial-phase hypoenhancing appearance on CT or MRI [Figure 4] ${ }^{[33]}$. On the other hand, HCCs typically show late (> $60 \mathrm{~s}$ after contrast injection) and mild washout ${ }^{[31,32]}$.

\section{The LI-RADS approach on CEUS}

The LI-RADS step-wise algorithmic approach on CEUS mirrors that of CT/MRI. Similar to CT/MRI, the LR-M categorization is an early step in the process intended to identify malignant nodules that may not be HCC. CEUS LR-M criteria include: rim-APHE, early (<60 s) washout, or marked washout visible within the first $120 \mathrm{~s}$ [Figures 4 and 5] ${ }^{[34,35]}$. The CEUS LI-RADS M criteria, like CT/MRI LR-M, reflect the imaging appearance of iCCA on CEUS.

CEUS LR-M criteria differ from CT/MRI LR-M criteria mostly due to the different properties of the contrast agents ${ }^{[33,36]}$. Figure 6 compares the criteria for LR-M and LR- 5 between CT/MRI and CEUS. Microbubble 

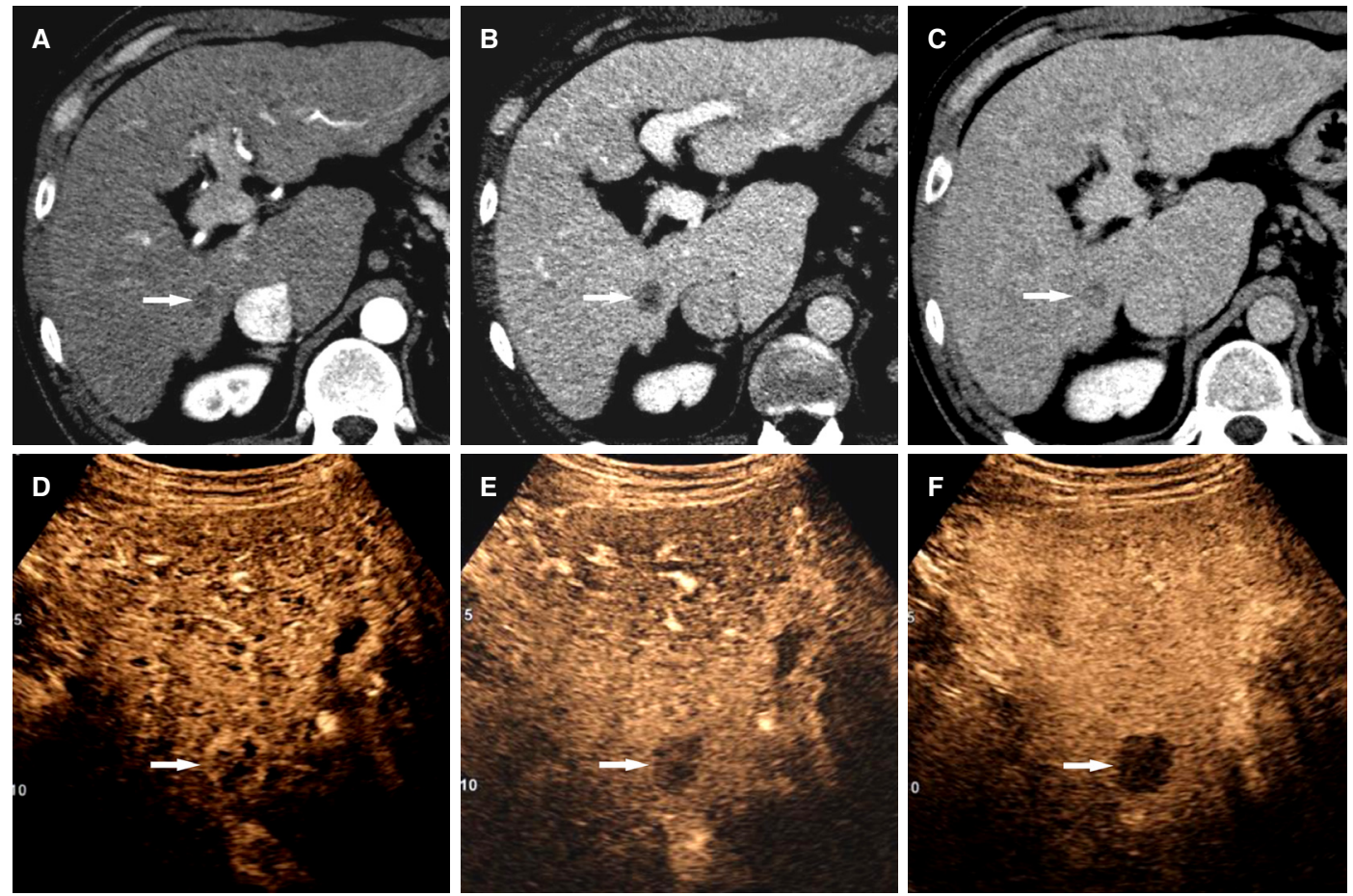

Figure 4. Intrahepatic cholangiocarcinoma in a 51-year-old man with hepatitis-C related liver cirrhosis. CT scans in the arterial (A), portal venous (B), and delayed (C) phases demonstrate a hypoattenuating hepatic nodule (arrow); C: the nodule shows a mild central enhancement in the delayed phase; D: CEUS in the arterial phase shows a rim arterial-phase hyperenhancement in the nodule (arrow); E: CEUS at $42 \mathrm{~s}$ after contrast injection demonstrates early washout (arrow); F: CEUS at $104 \mathrm{~s}$ after contrast injection shows marked washout (arrow). CEUS: contrast enhanced ultrasound
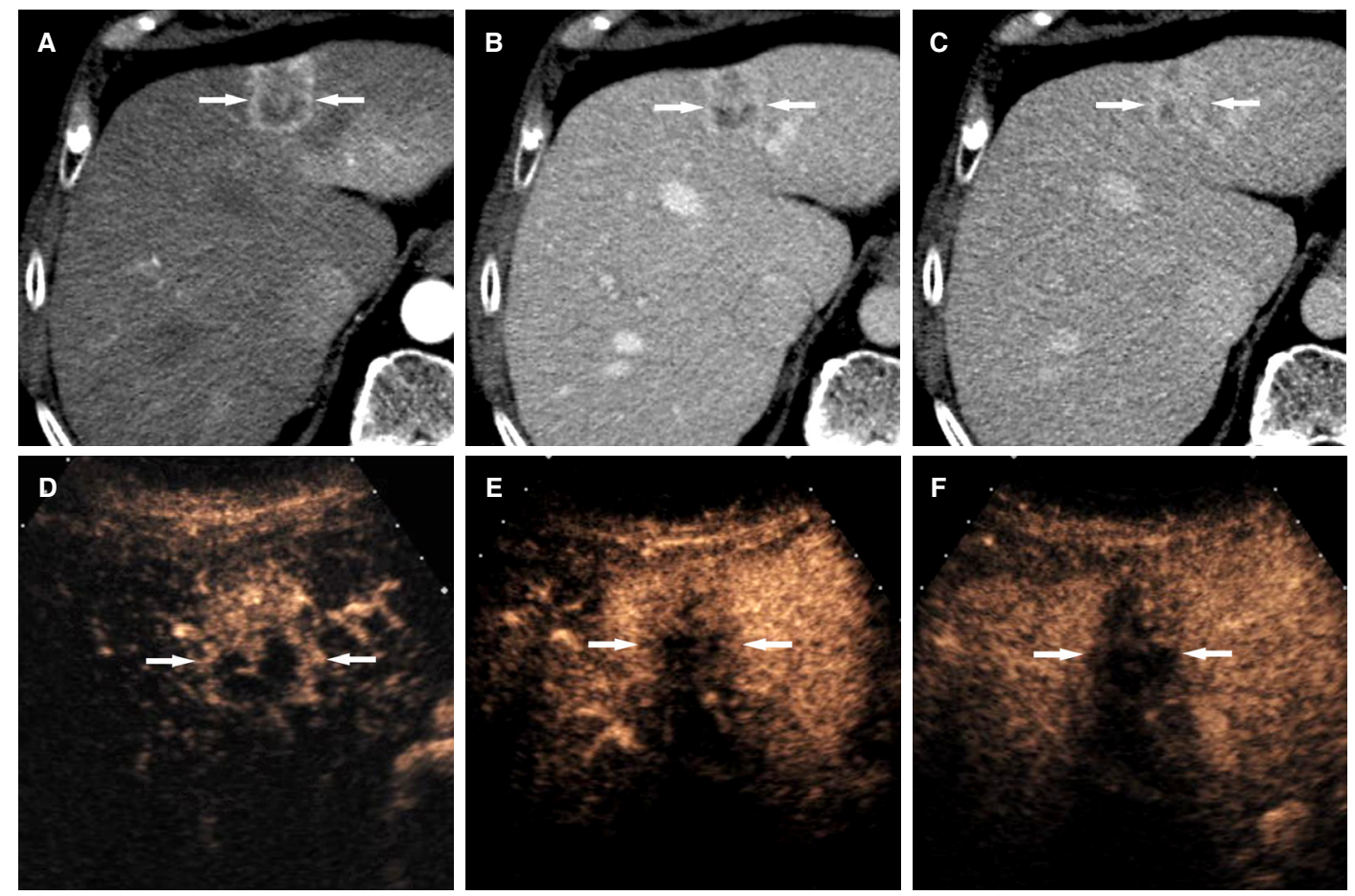

Figure 5. Intrahepatic cholangiocarcinoma in a 78-year-old man with hepatitis-C related liver cirrhosis. A: CT scans in the arterial phase shows a mass with rim arterial-phase hyperenhancement (arrows); CT scans in the portal venous (B), and delayed (C) phases demonstrates gradual enhancement of the mass (arrows); C: there is also heterogeneous hyperenhancement in the delayed phase; D: CEUS in the arterial phase shows irregular rim arterial-phase hyperenhancement in the mass (arrows); E: CEUS at $48 \mathrm{~s}$ after contrast injection demonstrates early washout (arrows); F: CEUS at $115 \mathrm{~s}$ shows marked washout (arrows). CEUS: contrast enhanced ultrasound 


\section{LR-M}
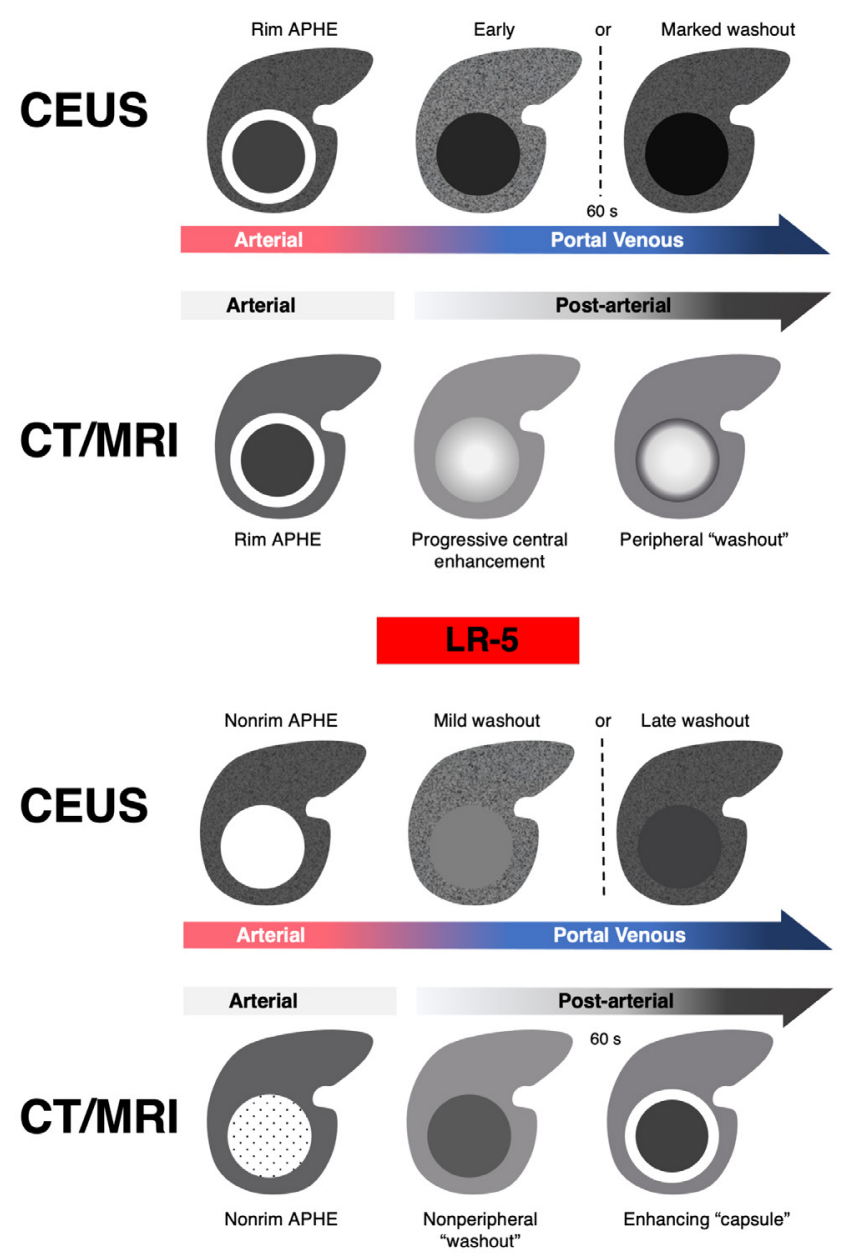

Figure 6. Schematics illustrating imaging features of LR-M and major imaging features of LR-5 according to CEUS LI-RADS and CT/MRI LI-RADS. CEUS: contrast enhanced ultrasound; LI-RADS: Liver Imaging Reporting and Data System; APHE: arterial phase hyperenhancement

contrast agents in CEUS are strictly intravascular because the large size of microbubbles does not permit them to pass through the vascular endothelium into the interstitial space, whereas the small molecules of CT/MRI contrast agents can pass through vascular endothelium. This difference in distribution of contrast translates to differences in imaging appearance. On CT/MRI, iCCAs typically demonstrate a gradual enhancement over time and often show delayed central enhancement due to hyperpermeability of the vascular endothelium and tumor interstitial constituents like necrosis or fibrosis that expand the extracellular space [Figure 5]. In contradistinction, the concentration of the microbubbles is uniformly distributed in the blood pool in the late phase and the degree of enhancement in the late phase mainly reflects the vascular volume on CEUS. The vascular volume in iCCA with fibrosis and absence of sinusoid-like structures is particularly low. Therefore, iCCAs invariably show marked washout in the late phase ${ }^{[37]}$.

\section{How accurate is CEUS LR-M for differentiating iCCA from HCC?}

The main role of the LR-M category is to prevent misdiagnosis of non-HCC malignancy as HCC. Therefore, the LR-M category should have a high sensitivity to diagnose non-HCC malignancy. In a large retrospective study including 1006 hepatic nodules by Terzi et al. ${ }^{[38]}$, 31/40 iCCAs were categorized as LR-M. The remaining 9/40 iCCAs were categorized as LR-3 or LR-4. No iCCAs were categorized as LR-5. However, it 

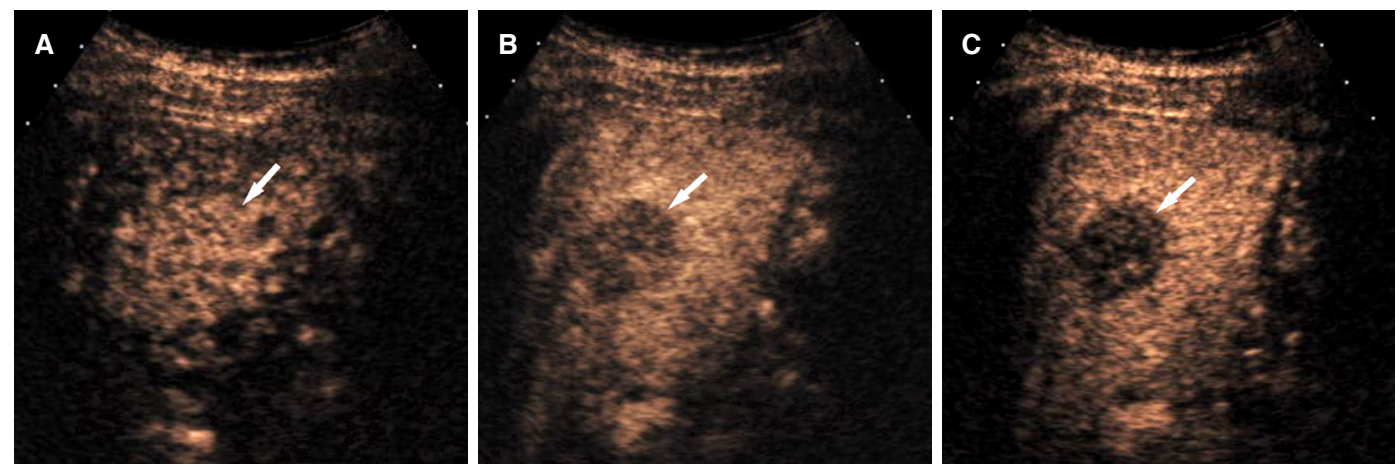

Figure 7. Combined hepatocellular-cholangiocarcinoma in a 77-year-old man with hepatitis-B related liver cirrhosis. A: contrast-enhanced CT or MRI could not be performed due to renal failure. CEUS in the arterial phase shows a mass with homogeneous arterial phase hyperenhancement (arrow); B: CEUS at $31 \mathrm{~s}$ after contrast injection shows early washout (arrow); C: CEUS at $60 \mathrm{~s}$ after contrast injection shows marked washout (arrow). CEUS: contrast enhanced ultrasound

is important to note that $48 \%$ (39/82) of LR-M observations were $\mathrm{HCCs}^{[38]}$. Later studies also confirmed the high sensitivity of CEUS LR-M criteria for diagnosing non-HCC malignancies ${ }^{[39-41]}$.

\section{MANAGEMENT CONSIDERATIONS}

Histologic diagnosis by biopsy is usually recommended for LR-M lesions. This is both to direct care for non-HCC malignancies and to identify the significant minority of atypical HCCs that are categorized as LR-M observations ${ }^{[17,18]}$. While retrospective data suggest that LR-M carries prognostic significance in the context of HCC, there is no prospective data to guide management decisions for these radiology-pathology discordant lesions. As of now, in the United States and Canada, if a lesion is biopsy proven to be HCC, it may be considered for standard HCC therapies including transplantation, despite its LR-M categorization. With additional data to support the prognostic significance of LR-M categorization, radiological appearances may be incorporated into future treatment algorithms for atypical HCC.

\section{Challenges and considerations-combined tumors}

While LI-RADS accurately differentiates HCC from iCCA, combined tumors (cHCC-CCA) still present a challenge. Combined hepatocellular cholangiocarcinomas are relatively rare primary liver carcinomas and as a result, the true epidemiology and risk factors are less well known. Several studies have shown some overlap in the risk factor profile for $\mathrm{CHCC}-\mathrm{CCA}$ and $\mathrm{HCC}^{[42]}$. In the latest World Health Organization classification of tumors 5th edition, cHCC-CCA are a distinct entity defined by the unequivocal presence of both hepatocytic and cholangiocytic differentiation within the same tumor ${ }^{[43]}$. In a retrospective review of 3103 adult liver transplantation from database entries by Jung et al. ${ }^{[44]}$, cHCC-CCA constituted $2.7 \%(32 / 1173)$ of patients with primary liver malignancies.

Imaging diagnosis is challenging because cHCC-CCA is not a homogeneous tumor. On CT/MRI, cHCCCCA appear to have more similarity with iCCA than HCC, often showing targetoid features ${ }^{[4,45,46]}$. However, there may be overlap in appearance with HCC in a minority of cases ${ }^{[4,45]}$. CEUS findings inevitably overlap with HCC and iCCA, depending on the amount of each component ${ }^{[47]}$. In a recent study by Zheng et al. ${ }^{[48]}$, $20 / 24(83 \%)$ of cHCC-CCA were categorized as LR-M on CEUS [Figure 7] and the remaining 4/24 (17\%) as LR-5.

\section{Challenges and considerations-reader agreement}

The main philosophy of LI-RADS is to preserve high specificity/PPV for the diagnosis of HCC. LI-RADS suggests that the major features of HCC be applied only if unequivocally present, and that when considering whether a feature represents a major vs. LR-M feature, it should be classified as LR-M. For instance, if in 
doubt about whether CEUS washout is marked or mild, it should be classified as marked. Likewise, if in doubt about rim APHE vs. nonrim APHE, we should call it rim APHE. These rules help direct individuals in the application of the LI-RADS algorithm, but do not necessarily address inter-reader variability, which is invariably encountered in clinical practice. While the literature suggests that agreement is good to excellent for LR-M vs. LR-5 categorization ${ }^{[4,49]}$, its reliability in routine practice is not well known and this remains an area of continued research and improvement for LI-RADS.

\section{FUTURE DIRECTIONS}

The prognostic value of LR features and categories is an area of active research with opportunities to investigate relationships of features with molecular profiles, immune landscapes, and histological subtypes of HCC that may inform individualized therapy.

As radiology continues to evolve toward big data and natural language processing applications, the LI-RADS categorical approach may be replaced or augmented by radiomics or deep learning models that have the potential to provide a more granular probability of diagnosing iCCA, cHCC-CCA, and HCC.

CEUS LI-RADS currently only addresses contrast agents that are FDA approved in the United States. Future versions may incorporate hepatocyte specific agents.

\section{CONCLUSION}

LI-RADS LR-M category is intended to capture all non-HCC malignancies. The criteria differs depending on modality. On CT/MRI, targetoid dynamic enhancement, diffusion weight, and hepatobiliary phase imaging are the primary features of LR-M. On CEUS, the presence of rim APHE and marked or early washout are the primary features of LR-M. When applied, LR-M criteria accurately capture almost all non-HCC malignancies and some atypical HCCs.

\section{DECLARATIONS}

\section{Authors' contributions}

Made substantial contributions to the conception and design of the study and performed data analysis and interpretation: Fowler KJ, Cunha GM, Kim TK

\section{Availability of data and materials}

Not applicable.

\section{Financial support and sponsorship}

None.

\section{Conflicts of interest}

Dr. Fowler KJ receives research support from Bayer and General Electric (GE healthcare) and from Innovis, Medscape and Bayer for consulting. Dr. Cunha GM and Dr. Kim TK declared that there are no conflict of interest with regard to this manuscript.

\section{Ethical approval and consent to participate}

Not applicable.

\section{Consent for publication}

Not applicable. 


\section{Copyright}

(C) The Author(s) 2020.

\section{REFERENCES}

1. El-Serag HB, Engels EA, Landgren O, Chiao E, Henderson L, et al. Risk of hepatobiliary and pancreatic cancers after hepatitis C virus infection: a population-based study of U.S. veterans. Hepatology 2009;49:116-23.

2. Yamamoto S, Kubo S, Hai S, Uenishi T, Yamamoto T, et al. Hepatitis C virus infection as a likely etiology of intrahepatic cholangiocarcinoma. Cancer Sci 2004;95:592-5.

3. Chernyak V, Fowler KJ, Kamaya A, Kielar AZ, Elsayes KM, et al. Liver Imaging Reporting and Data System (LI-RADS) Version 2018: imaging of hepatocellular carcinoma in at-risk patients. Radiology 2018;289:816-30.

4. Fraum TJ, Tsai R, Rohe E, Ludwig DR, Salter A, et al. Differentiation of hepatocellular carcinoma from other hepatic malignancies in patients at risk: diagnostic performance of the liver imaging reporting and data system version 2014. Radiology 2018;286:158-72.

5. Tang A, Bashir MR, Corwin MT, Cruite I, Dietrich CF, et al. Evidence supporting LI-RADS major features for CT- and MR imagingbased diagnosis of hepatocellular carcinoma: a systematic review. Radiology 2018;286:29-48.

6. Kang Y, Lee JM, Kim SH, Han JK, Choi BI. Intrahepatic mass-forming cholangiocarcinoma: enhancement patterns on gadoxetic acidenhanced MR images. Radiology 2012;264:751-60.

7. Peporte AR, Sommer WH, Nikolaou K, Reiser MF, Zech CJ. Imaging features of intrahepatic cholangiocarcinoma in Gd-EOB-DTPAenhanced MRI. Eur J Radiol 2013;82:e101-6.

8. Hwang J, Kim YK, Park MJ, Lee MH, Kim SH, et al. Differentiating combined hepatocellular and cholangiocarcinoma from massforming intrahepatic cholangiocarcinoma using gadoxetic acid-enhanced MRI. J Magn Reson Imaging 2012;36:881-9.

9. Chong YS, Kim YK, Lee MW, Kim SH, Lee WJ, et al. Differentiating mass-forming intrahepatic cholangiocarcinoma from atypical hepatocellular carcinoma using gadoxetic acid-enhanced MRI. Clin Radiol 2012;67:766-73.

10. Park HJ, Kim YK, Park MJ, Lee WJ. Small intrahepatic mass-forming cholangiocarcinoma: target sign on diffusion-weighted imaging for differentiation from hepatocellular carcinoma. Abdom Imaging 2013;38:793-801.

11. Rimola J, Forner A, Reig M, Vilana R, de Lope CR, et al. Cholangiocarcinoma in cirrhosis: absence of contrast washout in delayed phases by magnetic resonance imaging avoids misdiagnosis of hepatocellular carcinoma. Hepatology 2009;50:791-8.

12. Kim SJ, Lee JM, Han JK, Kim KH, Lee JY, et al. Peripheral mass-forming cholangiocarcinoma in cirrhotic liver. AJR Am J Roentgenol 2007;189:1428-34.

13. Nakanuma Y, Komuta M, Zen Y. WHO Classification of tumors. Digestive System Tumors. 5th ed. Lyon (France): International Agency for Research on Cancer; 2019.

14. Fraum TJ, Cannella R, Ludwig DR, Tsai R, Naeem M, et al. Assessment of primary liver carcinomas other than hepatocellular carcinoma (HCC) with LI-RADS v2018: comparison of the LI-RADS target population to patients without LI-RADS-defined HCC risk factors. Eur Radiol 2020;30:996-1007.

15. Fowler KJ, Potretzke TA, Hope TA, Costa EA, Wilson SR. LI-RADS M (LR-M): definite or probable malignancy, not specific for hepatocellular carcinoma. Abdom Radiol (NY) 2018;43:149-57.

16. Horvat N, Nikolovski I, Long N, Gerst S, Zheng J, et al. Imaging features of hepatocellular carcinoma compared to intrahepatic cholangiocarcinoma and combined tumor on MRI using liver imaging and data system (LI-RADS) version 2014. Abdom Radiol (NY) 2018;43:169-78.

17. Ludwig DR, Fraum TJ, Cannella R, Ballard DH, Tsai R, et al. Hepatocellular carcinoma (HCC) versus non-HCC: accuracy and reliability of Liver Imaging Reporting and Data System v2018. Abdom Radiol (NY) 2019;44:2116-32.

18. Shao S, Liang Y, Kuang S, Chen J, Shan Q, et al. Diagnostic performance of LI-RADS version 2018 in differentiating hepatocellular carcinoma from other hepatic malignancies in patients with hepatitis B virus infection. Bosn J Basic Med Sci 2020; Epub ahead of print. doi: $10.17305 /$ bjbms.2019.4576.

19. Lee HS, Kim MJ, An C. How to utilize LR-M features of the LI-RADS to improve the diagnosis of combined hepatocellularcholangiocarcinoma on gadoxetate-enhanced MRI? Eur Radiol 2019;29:2408-16.

20. van der Pol CB, Lim CS, Sirlin CB, McGrath TA, Salameh JP, et al. Accuracy of the liver imaging reporting and data system in computed tomography and magnetic resonance image analysis of hepatocellular carcinoma or overall malignancy-a systematic review. Gastroenterology 2019;156:976-86.

21. Park YS, Lee CH, Kim BH, Lee J, Choi JW, et al. Using Gd-EOB-DTPA-enhanced 3-T MRI for the differentiation of infiltrative hepatocellular carcinoma and focal confluent fibrosis in liver cirrhosis. Magn Reson Imaging 2013;31:1137-42.

22. Lim S, Kim YK, Park HJ, Lee WJ, Choi D, et al. Infiltrative hepatocellular carcinoma on gadoxetic acid-enhanced and diffusion-weighted MRI at 3.0T. J Magn Reson Imaging 2014;39:1238-45.

23. Choi SY, Kim YK, Min JH, Kang TW, Jeong WK, et al. Added value of ancillary imaging features for differentiating scirrhous hepatocellular carcinoma from intrahepatic cholangiocarcinoma on gadoxetic acid-enhanced MR imaging. Eur Radiol 2018;28:2549-60.

24. Seo N, Kim MJ, Rhee H. Hepatic sarcomatoid carcinoma: magnetic resonance imaging evaluation by using the liver imaging reporting and data system. Eur Radiol 2019;29:3761-71.

25. Choi SH, Lee SS, Park SH, Kim KM, Yu E, et al. LI-RADS Classification and prognosis of primary liver cancers at gadoxetic acidenhanced MRI. Radiology 2019;290:388-97.

26. Vilana R, Forner A, Bianchi L, Garcia-Criado A, Rimola J, et al. Intrahepatic peripheral cholangiocarcinoma in cirrhosis patients may 
display a vascular pattern similar to hepatocellular carcinoma on contrast-enhanced ultrasound. Hepatology 2010;51:2020-2029.

27. Wildner D, Bernatik T, Greis C, Seitz K, Neurath MF, et al. CEUS in hepatocellular carcinoma and intrahepatic cholangiocellular carcinoma in 320 patients - early or late washout matters: a subanalysis of the DEGUM multicenter trial. Ultraschall Med 2015;36:132-9.

28. Bhayana D, Kim TK, Jang HJ, Burns PN, Wilson SR. Hypervascular liver masses on contrast-enhanced ultrasound: the importance of washout. AJR Am J Roentgenol 2010;194:977-83.

29. Han J, Liu Y, Han F, Li Q, Yan C, et al. The degree of contrast washout on contrast-enhanced ultrasound in distinguishing intrahepatic cholangiocarcinoma from hepatocellular carcinoma. Ultrasound Med Biol 2015;41:3088-95.

30. Li R, Yuan MX, Ma KS, Li XW, Tang CL, et al. Detailed analysis of temporal features on contrast enhanced ultrasound may help differentiate intrahepatic cholangiocarcinoma from hepatocellular carcinoma in cirrhosis. PLoS One 2014;9:e98612.

31. Jang HJ, Kim TK, Burns PN, Wilson SR. Enhancement patterns of hepatocellular carcinoma at contrast-enhanced US: comparison with histologic differentiation. Radiology 2007;244:898-906.

32. Jang HJ, Kim TK, Burns PN, Wilson SR. CEUS: an essential component in a multimodality approach to small nodules in patients at highrisk for hepatocellular carcinoma. Eur J Radiol 2015;84:1623-35.

33. Wilson SR, Kim TK, Jang HJ, Burns PN. Enhancement patterns of focal liver masses: discordance between contrast-enhanced sonography and contrast-enhanced CT and MRI. AJR Am J Roentgenol 2007;189:W7-12.

34. Wilson SR, Lyshchik A, Piscaglia F, Cosgrove D, Jang HJ, et al. CEUS LI-RADS: algorithm, implementation, and key differences from CT/MRI. Abdom Radiol (NY) 2018;43:127-42.

35. Lyshchik A, Kono Y, Dietrich CF, Jang HJ, Kim TK, et al. Contrast-enhanced ultrasound of the liver: technical and lexicon recommendations from the ACR CEUS LI-RADS working group. Abdom Radiol (NY) 2018;43:861-79.

36. Kim TK, Noh SY, Wilson SR, Kono Y, Piscaglia F, et al. Contrast-enhanced ultrasound (CEUS) liver imaging reporting and data system (LI-RADS) 2017 - a review of important differences compared to the CT/MRI system. Clin Mol Hepatol 2017;23:280-9.

37. Yang HK, Burns PN, Jang HJ, Kono Y, Khalili K, et al. Contrast-enhanced ultrasound approach to the diagnosis of focal liver lesions: the importance of washout. Ultrasonography 2019;38:289-301.

38. Terzi E, Iavarone M, Pompili M, Veronese L, Cabibbo G, et al. Contrast ultrasound LI-RADS LR-5 identifies hepatocellular carcinoma in cirrhosis in a multicenter restropective study of 1,006 nodules. J Hepatol 2018;68:485-92.

39. Li F, Li Q, Liu Y, Han J, Zheng W, et al. Distinguishing intrahepatic cholangiocarcinoma from hepatocellular carcinoma in patients with and without risks: the evaluation of the LR-M criteria of contrast-enhanced ultrasound liver imaging reporting and data system version 2017. Eur Radiol 2020;30:461-70.

40. Chen LD, Ruan SM, Liang JY, Yang Z, Shen SL, et al. Differentiation of intrahepatic cholangiocarcinoma from hepatocellular carcinoma in high-risk patients: A predictive model using contrast-enhanced ultrasound. World J Gastroenterol 2018;24:3786-98.

41. Chen LD, Ruan SM, Lin Y, Liang JY, Shen SL, et al. Comparison between M-score and LR-M in the reporting system of contrastenhanced ultrasound LI-RADS. Eur Radiol 2019;29:4249-57.

42. Brunt E, Aishima S, Clavien PA, Fowler K, Goodman Z, Gores G, Gouw A, et al. cHCC-CCA: Consensus terminology for primary liver carcinomas with both hepatocytic and cholangiocytic differentation. Hepatology 2018;68:113-126.

43. Sciarra A, Park YN, Sempoux C. Updates in the diagnosis of combined hepatocellular-cholangiocarcinoma. Hum Pathol 2020;96:48-55.

44. Jung DH, Hwang S, Song GW, Ahn CS, Moon DB, et al. Longterm prognosis of combined hepatocellular carcinoma-cholangiocarcinoma following liver transplantation and resection. Liver Transpl 2017;23:330-41.

45. Potretzke TA, Tan BR, Doyle MB, Brunt EM, Heiken JP, et al. Imaging features of biphenotypic primary liver carcinoma (Hepatocholangiocarcinoma) and the potential to mimic hepatocellular carcinoma: LI-RADS analysis of CT and MRI features in 61 Cases. AJR Am J Roentgenol 2016;207:25-31.

46. Wells ML, Venkatesh SK, Chandan VS, Fidler JL, Fletcher JG, et al. Biphenotypic hepatic tumors: imaging findings and review of literature. Abdom Imaging 2015;40:2293-305.

47. Li F, Han J, Han F, Wang JW, Luo RZ, et al. Combined hepatocellular cholangiocarcinoma (Biphenotypic) tumors: potential role of contrast-enhanced ultrasound in diagnosis. AJR Am J Roentgenol 2017;209:767-74.

48. Zheng W, Li Q, Zou XB, Wang JW, Han F, et al. Evaluation of Contrast-enhanced US LI-RADS version 2017: application on 2020 liver nodules in patients with hepatitis B infection. Radiology 2020;294:299-307.

49. Abdel Razek AAK, El-Serougy LG, Saleh GA, Abd El-Wahab R, Shabana W. Interobserver agreement of magnetic resonance imaging of liver imaging reporting and data system version 2018. J Comput Assist Tomogr 2020;44:118-23. 